\title{
Hierarchical Hexagonal Modulation with Ternary Symbols for Wireless Video Transmission
}

\author{
Zhe Yang*, Lin Cai, Xuan Wang, Siyuan Xiang, and Jianping Pan \\ *Northwest Polytechnical University, Xi' an, Shaanxi, China \\ University of Victoria, Victoria, BC, Canada
}

\begin{abstract}
Wireless video transmission systems have been widely adopted to facilitate the ubiquitous access of the multimedia services on the Internet. However, wireless links are not stable and the channel quality might vary with time. Efficient modulation scheme plays an important role to improve the quality of service (QoS) of video transmission. However, the previous efforts mainly focused on the rectangular constellation to carry coded information bits. In this paper, we go beyond the existing research efforts and bring non-binary communication and the hexagonal modulation together with a hybrid structure which can be compatible with the existing systems, and show by both prototyping and simulation their efficacy in wireless communication systems with varying channel conditions. For video applications requiring differentiated services, we further design hierarchical hexagonal modulation. The new cross-layer approach offers a new dimension in the pursuit of squeezing more out of the limited energy and spectrum resources in supporting wireless video applications.
\end{abstract}

Index Terms-Hexagonal modulation, video transmission, differentiated services

\section{INTRODUCTION}

According to Cisco Visual Networking Index studies, twothirds of the world's mobile data traffic will be video by 2016 [1]. As the last meter/mile solution to support the increasing demand, wireless communication is an essential component of the modern information system. Given the scarcity of wireless spectrum, how to support video efficiently with satisfactory quality of services (QoS), however, is the grand challenge for both the research community and industry.

In the wireless domain, given channel dynamics and the high cost of accurate channel estimation, a promising solution is to provide differentiated services for data with different importance, using hierarchical modulation (HM) [2]-[4]. The existing HM approaches are based on square quadratic modulation (QAM). It is already known in two-dimensional regular tiling that hexagons, instead of squares, are the most efficient packing approach in terms of their compactness. Historically, however, hexagonal modulation (H-QAM) has not been widely used because of several implementation issues that wipe away the gain by H-QAM.

By investigating the limitation of the existing implementation of H-QAM, we take the liberty of adopting ternary digits (trits) to carry information during transmission, which can take the best usage of H-QAM. We further design new hierarchical H-QAM for providing service differentiation, which is desirable for supporting video applications.
Our main contributions in this paper are highlighted as follows. We design new, hierarchical H-QAM constellation that can not only improve the spectrum and energy efficiency, but also provide differentiated services for video applications to enhance the perceived video quality. Extensive trace-driven simulations have been conducted, and the results have shown the substantial performance gain of the proposed hierarchical H-QAM solution.

In summary, this paper shows the efficacy of the hierarchical H-QAM approach with non-binary information mapping for supporting video over wireless networks. The implementation of the proposed hierarchical H-QAM and ternary symbol involves a simple upgrade to the modulation and coding module in the communication system only, so the solution is feasible to be incrementally deployed.

The rest of the paper is organized as follows. In Section II, we discuss the background information and the related work. In Section III, we present our design motivation and the prototype results. In Section IV, we give the detailed design of a non-binary hierarchical H-QAM communication system, including the hexagonal symbol constellation structure, information-to-symbol mapping, non-binary error coding and interleaving, H-QAM configuration, and the packetization interface with the upper layers. In Section V, we investigate the system performance through extensive trace-driven simulation, followed by the concluding remarks and further research issues in Section VI.

\section{BACKGROUND AND RELATED WORK}

\section{A. Hexagonal Constellation}

For the sake of clarity, we list the important terms used in the paper and their meanings or examples in Table 1.

The previous work on hexagonal modulation often stuck to the binary representation and the number of constellation points is a power of two. Therefore, they need to omit some of the constellation points in the hexagonal constellation [5].

Another scheme that combines the non-binary coding with hexagonal modulation has been adopted in [6]. It can fully utilize the constellation points of H-QAM by mixing ternary symbols and binary symbols. However, all the transmitted information is treated equally. In this paper, we take one-step further. We not only use ternary error coding to protect trits directly, but also design new hierarchical H-QAM constellation that not existed in the literature. 


\begin{tabular}{|l|l|}
\hline Terms & Meanings/Examples \\
\hline Trit & Ternary digit ("0", "1", or "2"), a trit contains $\log _{2} 3$ bits of information \\
Constellation arrangements & Geometrically locate constellation points in the signal space for modulation \\
Traditional modulation schemes & BPSK, QPSK, 16-QAM, etc. \\
Hexagonal modulation schemes & TPSK, H6-QAM, H8-QAM, H12-QAM, etc. \\
Hierarchical modulation schemes & each symbol carries bits/trits with different error performance \\
Constellation mapping & Mapping from bits and/or trits to symbols (Gray code, etc.) \\
\hline
\end{tabular}

TABLE I

SOME TERMINOLOGIES USED FOR DIFFERENT MECHANISMS DISCUSSED IN THIS PAPER.

\section{B. Hierarchical Modulation for Service Differentiation}

For video applications, data belong to different frames (e.g., I/B/P frames in MPEG-like codecs) or different layers have different sensitivities to loss, and it is essential to provide differentiated services (DiffServ) for them [7]. Providing DiffServ at the physical layer has the advantage of easy synchronization and high robustness against varying wireless channel conditions: multiplexing the information from different layers and transmitting them in the same symbol, different layers of the same frame can be received simultaneously. When the wireless channel quality is good, all layers can be received successfully,otherwise at least the most important layers can be received successfully.

Back to 1970's, Cover had suggested that, for a broadcast channel, by super-imposing signals and transmitting them simultaneously to multiple users with different channel quality, a higher throughput can be achieved. This idea has been adopted by several industry standards (such as DVB-T, MediaFLO, UMB-BCMCS) and the practical implementation is called hierarchical modulation (HM). Using HM, a single symbol can be mapped to bits with different error performance or SNR requirements. HM offers several benefits for both video multicast or unicast in wireless environment. Using HM, important video data (such as the base layer in SVC) can be transmitted with lower SNR requirements, so the majority of the users can receive them successfully; the less-important data (such as the enhancement layer) can be transmitted with higher SNR requirements, so only the users with good channel can decode them successfully to enjoy a higher video quality.

Comparing HM with the strategy of using different types of modulations for different video layers, HM is more flexible in making a tradeoff between the two layers. For instance, in a fading channel, if the base layer and enhancement layer of the same frame are transmitted separately, it is possible that an enhancement layer is received successfully but useless, as its corresponding base layer is lost.

\section{Prototy ping Hexagonal Modulation}

To prove the feasibility of the ternary H-QAM communication system, we used the software defined radio (SDR) platform USRP2 and GNU Radio to prove the concept [8]. We used the XCV2450 daughter board which can be operated at the license-free bands of 2.4 to $2.5 \mathrm{GHz}$ and 4.9 to $5.85 \mathrm{GHz}$. We connected one USRP2 to the laptop host (DELL E5400) as the transmitter and another one to the PC host (DELL OPTIPLEX 755) as the receiver. As OFDM has been a

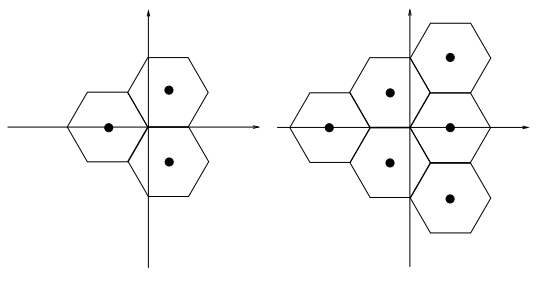

(a)

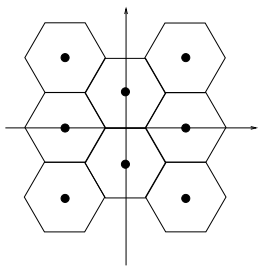

(c)
Fig. 1. TPSK, H6-QAM, and H8-QAM Constellation.

\begin{tabular}{|l|l|l|}
\hline Modulation & Raw BER & Coded BER \\
\hline BPSK & $5.4 \times 10^{-5}$ & $<10^{-7}$ \\
\hline TPSK & $5.1 \times 10^{-5}$ & $<10^{-7}$ \\
\hline QPSK & $2.9 \times 10^{-4}$ & $2.13 \times 10^{-7}$ \\
\hline H6-QAM & $1.2 \times 10^{-3}$ & $5.49 \times 10^{-6}$ \\
\hline H8-QAM & $1.6 \times 10^{-3}$ & $1.04 \times 10^{-5}$ \\
\hline 8PSK & $2.3 \times 10^{-3}$ & $1.22 \times 10^{-5}$ \\
\hline
\end{tabular}

TABLE II

ERror Rate MEASUREMEnt RESUlts.

popular component of many modern communication systems, our prototype is also based on the OFDM technology. We augmented the USRP2-based OFDM implementation [9] by adopting the proposed H-QAMs. We set the carrier frequency, the number of subcarriers and the FFT length to be $2.49 \mathrm{GHz}$, 80 and 512 , respectively.

We implemented three hexagonal modulation designs, ternary phase shifted key (TPSK), H6-QAM and H8-QAM. The constellations of these modulations are shown in Fig. 1. Other H-QAM designs can be implemented using a similar approach. To be compatible to the conventional binary communication and computing systems, the bits/trits conversion is necessary to utilize TPSK or other H-QAM schemes. An efficient and low cost approach is to convert 11 bits to 7 trits, yielding an efficiency of $\frac{11 \log _{2} 2}{7 \log _{2} 3}=99.1 \%$ [10]. Thus, we convert each 11-bit data block to a 7-trit block. As the block length is short, the conversions to/from trits can be conducted quickly using a lookup table.

We used bit error rate (BER) as the metric to compare the performance of H-QAMs and the conventional modulations. We let the transmitter consecutively transmit 1,000 test frames. Each frame contains 100 blocks of data, and each block contains 16 bytes of data and 4 bytes of the CRC code. The transmitter converts all or part of the bits in each block to trits if we use TPSK or H6-QAM, respectively. The receiver demodulates the received symbol to bits or trits first, according to the modulation scheme used and indicated in the PLCP 
header. We compared the received data before error coding and the transmitted data to obtain the raw BER. To obtain the coded BER performance, the received bits or trits are further processed using a $1 / 2$ binary convolutional code and a $1 / 2$ ternary convolutional code, respectively.

The BER results are shown in Table II. In general, as expected, the BER of higher modulation is higher, and H8-QAM out-performs 8PSK thanks to its compact hexagonal structure. The prototype implementation demonstrates the feasibility and simplicity of using H-QAM and non-binary symbols.

Surprisingly, the BER performance of TPSK is even slightly better than that of BPSK under the same experimental condition. The causes of this observed phenomenon are still under investigation. We will leave this interesting counter intuitive observation to further research.

\section{HieRARCHICAL H-QAM DESIGN}

To fully utilize the most compact hexagonal constellation for modulation and to mix bits and trits for transmission, we need to redesign and re-investigate the following basic building blocks of a communication system: the modulation constellation geometry arrangement, the mapping to bits and trits, the error control coding with interleaver, and the packetization in multiplexing bits and trits.

\section{A. Constellation Geometry Arrangement}

1) Proposed Hierarchical H-QAM Geometry Arrangement: To support service differentiation, the hierarchical H-QAM (HH-QAM) design should maintain different error rates for different bits/trits. That is, the minimum Euclidean distance (MED) from the constellation points to the decision boundaries for different bit/trit should be set according to its priority. To achieve so, we can group the constellation points into clusters, and the MED for points within the cluster can be smaller than that for points belong to two different clusters ${ }^{1}$.

Guided by the densest packing, depending on the number of constellation points needed, we find the corresponding optimal H-QAM, with the minimum distance between two neighboring points equal to $2 d$. Then, depending on the number of $\mathrm{L} 1$ information needed, we group the constellation points into several clusters. Next, we move these clusters away from the origin such that the minimum distance between any two points belong to two different clusters is $2 D$.

For example, we use the above rule to design an $\mathrm{HH}$ 12QAM as shown in Fig. 2(a). The design is based on the constellation diagram of H12-QAM and the objective is to transmit one L1 trit and two L2 bits simultaneously. The 12 constellation points are grouped into three clusters. We separate the three clusters to ensure the distance between any two points of two clusters is at least $2 D$. When separating the clusters, it is important to ensure the "center of gravity" still collocates with the origin. To achieve so, we separate the two top clusters horizontally by $\left(\sqrt{4 D^{2}-d^{2}}-\sqrt{3 d^{2}}\right)$, move them up by $\frac{\sqrt{3}}{6}\left(\sqrt{4 D^{2}-d^{2}}-\sqrt{3 d^{2}}\right)$, and move the bottom cluster

\footnotetext{
${ }^{1}$ Note that, in this paper, we consider two layer HH-QAM, and how to extend the results to more layers is left for future research.
}

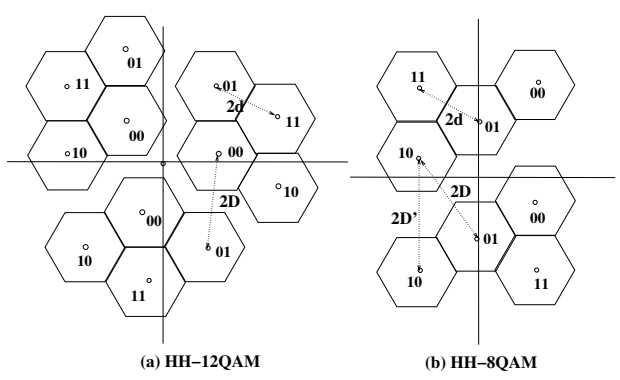

Fig. 2. Hierarchical H-QAM Constellations.

down by $\frac{\sqrt{3}}{3}\left(\sqrt{4 D^{2}-d^{2}}-\sqrt{3 d^{2}}\right)$. The average symbol energy equals $\frac{4}{3} D^{2}+3 d^{2}+\frac{2 d \sqrt{4 D^{2}-d^{2}}}{\sqrt{3}}$.

For easy comparison to the traditional QAM based hierarchical modulation, we also design an HH-8QAM as shown in Fig. 2 (b). We use H-8QAM as the basis, and separate the eight points into two clusters, and move them up or down by $D^{\prime}-d$, respectively, where $D^{\prime}=\sqrt{D^{2}-0.75 d^{2}}+0.5 d$. Again, the MED for the points belong to the same cluster is $2 d$, and that for points belong to different clusters is $2 D$. The average symbol energy equals $D^{\prime 2}+3 d^{2}+0.5 D^{\prime} d$.

Using the similar approach of HH-12QAM, we can design HH-6QAM or HH-9QAM to transmit one L1 trit and one L2 bit or trit. Given the space limitation, here we focus on the two HH-QAM proposed in Fig. 2 only, and investigate their performance by comparing with the existing solutions.

2) Constellation Mapping: Using the BER estimation above, to minimize the error rate, one key issue is to minimize the Hamming distance between neighboring points. As there are more neighboring points (with an equal distance to a constellation point) using H-QAM, without careful mapping, each symbol error will result in several bits and/or trits in error, so the BER performance will deteriorate. For a constellation size below 64 , it may not be possible to obtain the Gray-code type of mapping (to ensure only one bit/trit difference among neighboring points), because the number of neighbors with hexagonal tiling often exceeds the number of bits and trits represented by each symbol. Nevertheless, we can optimize the mapping by minimizing the average Hamming distance.

For the HH-12QAM, as shown in Fig. 2 (a), one trit is mapped for identifying the cluster. Within the cluster, we use the mapping similar to Gray-code, except that there are two neighboring points with a Hamming distance of two, because these points have three neighbors. For points closer to the origin, the same layer-two bits are used.

For HH-8QAM, the L1 bit is mapped to identify the two clusters. Within the cluster, we also minimize the Hamming distance between neighboring points, and the resultant mapping is shown in Fig. 2 (b).

3) HM configuration: The advantage of hierarchical modulation is the capability of adapting the two layer performance by adjusting the ratio of $D$ and $d$ according to their BER requirements and the estimated channel quality and channel profile. For an AWGN channel or a fading channel with known channel state information (CSI) (e.g., the receiver can feedback CSI to the sender promptly), given the received SNR, we can 


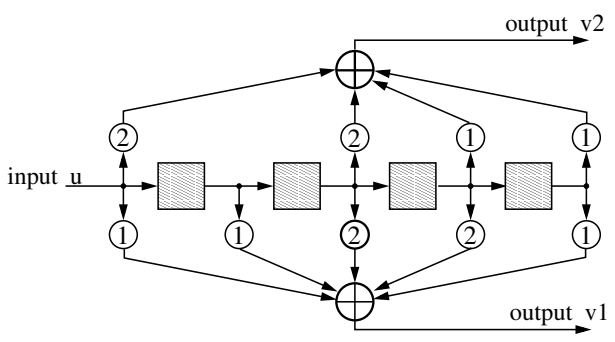

Fig. 3. Ternary Convolutional Encoder [12].

first determine $d$ to ensure L2 BER is satisfied. Then, the remaining energy can be used to transmit L1 bits/trits, and then we can calculate $e_{1}$. In case that the BER requirement of $\mathrm{L} 1$ cannot be satisfied, we need to select a modulation that contains fewer constellation points.

For a fast-fading channel without accurate CSI information, we should further consider the L1 error rate during the deep fading period and further enlarge the performance gap between the two layers, so the high priority information can be protected.

\section{B. Ternary Convolutional Code}

Convolutional code has been widely used thanks to its relatively simple implementation and the decent performance. A typical binary $(n, k, m)$ convolutional encoder can map $k$ input bits to $n$ output bits with a code rate of $k / n$ coded bitstream, and $m$ is the number of memory registers. Considering the implementation complexity, the Viterbi algorithm is the most popular maximum-likelihood decoding algorithm that has been widely adopted.

Similarly, a ternary $(n, k, m)$ convolutional encoder can map $k$ input trits to $n$ output trits with a code rate of $k / n$ [12]. A ternary convolutional encoder can be implemented by using a few shift registers and modulo-3 adders, as shown in Fig. 3. In the figure, the shaded squares are the shift registers, the circled " 1 " or " 2 " are the coefficients that will be multiplied to the input trits in the GF(3) field, and the circled sum represents a modulo-3 adder. As there are two output trit streams v1 and $v 2$, this convolutional code has a code rate of $1 / 2$, and it belongs to the non-recursive, non-systematic convolutional encoder category.

The decoder can also take the Viterbi algorithm, which takes $O\left(3^{m} L\right)$ memory space and $O\left(3^{m+1} L\right)$ computation time, where $L$ is the trace-back length of the decoder. The computation complexity of the ternary convolutional code can be comparable to that of the binary one, if the numbers of the states are in the same order.

\section{System Design and Implementation}

To support the proposed HH-QAM and ternary symbols, we next describe the new communication system design that can leverage the advantage of multiplexing bits and trits for wireless video applications.

Taking the IEEE 802.11a as an example, Fig. 4 shows the augmented structure to utilize the non-binary HH-QAMs. The

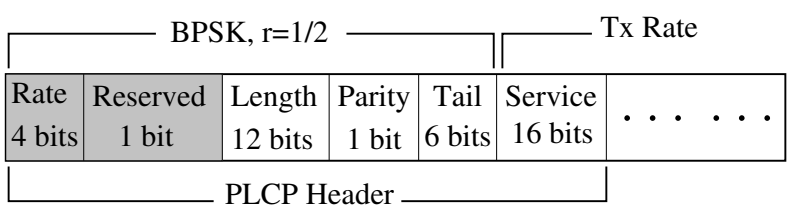

Fig. 5. PLCP Header with the Extended Rate Field.

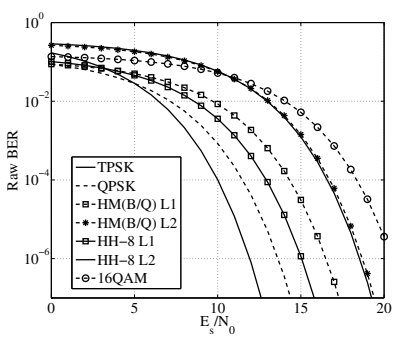

(a)

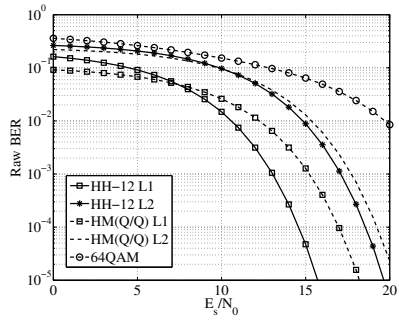

(b)
Fig. 6. BER Comparison, without Error Coding.

shaded areas are the new modules or the existing modules that need to be updated. For the application layer, we use scalable video streams as an example, in which there are two layers of information, the base layer (BL) and the enhancement layer (EL). The fragmentation module puts them into different queues as they will be processed differently. The bits/trits conversion module converts the bit sequence to a trit sequence, of which the details will be discussed later, and passes trits to the ternary-based convolutional coding (CC) encoder. The crosslayer controller takes the CSI to determine the modulation and coding schemes to be used, which is included in the Physical Layer Convergence Procedure (PLCP) header [13].

The PLCP header includes the control information in the physical layer. The rate field of the PLCP header shown in Figure 5 is used to inform the receiver the modulation and coding schemes. There are 4-bit field in the current $802.11 \mathrm{a}$ standard, which can be combined with another one reserved bit in the header shown as the shaded area in Fig. 5 to represent up to 32 different modulation and coding schemes. This should be sufficient to include all of the non-binary $\mathrm{HH}$ QAM schemes proposed in this paper. If in the future, finergrained of modulation schemes are adopted, at most a few more bits overhead is needed. Thus, the proposed non-binary, HH-QAM communication system can be easily adopted and backward compatible with the conventional ones.

\section{Performance Evaluation}

\section{A. Error Rate Comparison}

For modulation design, the key performance index is the raw $\mathrm{BER}^{2}$ given the received SNR over an AWGN channel. We let the received SNR vary from 0 to $20 \mathrm{~dB}$ to test the raw BER of different modulation schemes without error coding first. The results are shown in Fig. 6 (a) and (b), where the Xaxis represents the received symbol SNR (i.e., $E_{s} / N_{0}$ where $E_{s}$ is the received energy per symbol and $N_{0}$ is the noise

${ }^{2}$ For trits, at the receiver side, we convert them back to bits to calculate the BER. 


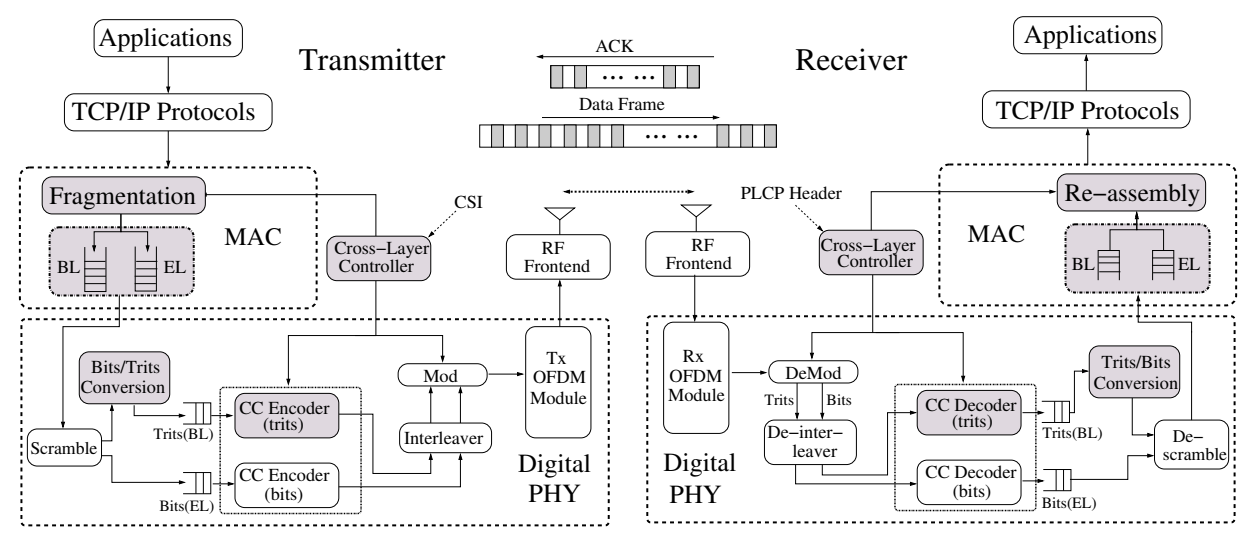

Fig. 4. System Design (Based on IEEE 802.11).

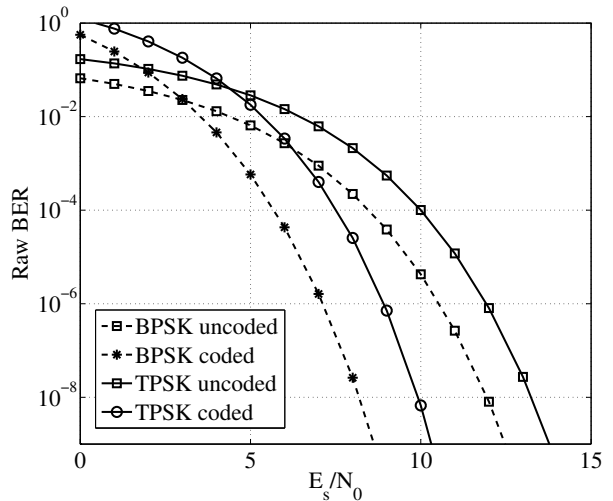

Fig. 7. Binary vs. Ternary Error Coding.

power spectral density) in $\mathrm{dB}$, and the $\mathrm{y}$-axis represents the raw BER in log-scale.

First, as anticipated, when we modulate more bits and trits in each symbol, the required SNR to reach a desired BER performance increases monotonically. However, by flexibly making a tradeoff between the two layers, for any given SNR, we can select and tune the HH-QAM to maximize the utility of the channel.

For instance, assume that the raw BER requirements for $\mathrm{L} 1$ and L2 are $10^{-4}$ and $10^{-3}$, respectively. When the SNR is between 12 and $17 \mathrm{~dB}$, using traditional modulation (without hierarchical), we can only use QPSK to support both L1 and L2, resulting in an average throughput of one L1 and one L2 bit/symbol. If using the proposed HH-8QAM, we can transmit one L1 and two L2 bit/symbol, a $50 \%$ throughput gain.

Second, comparing the proposed HH8-QAM and the existing $\mathrm{HM}$ (BPSK/QPSK), both support one L1 and two L2 bits per symbol, HH8-QAM can outperform the existing HM(BPSK/QPSK) by more than $1 \mathrm{~dB}$ for L1 when BER is below $10^{-3}$, while they have the same L2 performance. The results demonstrate the advantage of using hexagonal constellation.

Next, we investigate the performance of binary and ternary error codes. We used a convolutional encoder with rate $1 / 2$ for all the trits as shown in Fig. 3 in our simulation. For a fair comparison, we also used a binary convolutional encoder with rate $1 / 2$ for all the bits as well.

Figure 7 compares the uncoded and coded BER performance of BPSK with the binary CC coding and that of TPSK with ternary $\mathrm{CC}$ coding. From the figure, the ternary $\mathrm{CC}$ code has similar performance to the binary $\mathrm{CC}$ code. When the raw BER is $10^{-2}$ and $10^{-3}$, the ternary $\mathrm{CC}$ code can bring down the coded BER to $10^{-2.9}$ and $10^{-5.5}$, respectively, while the binary CC code achieves $10^{-2.7}$ and $10^{-5.6}$, respectively. Therefore, the coded BERs for bits and trits will be similar if their raw BERs are similar.

\section{B. Video Performance Evaluation}

1) Simulation Settings: We used a layered video as an example to demonstrate the advantages of HH-QAM. The video Foreman is used as the test video sequence. The video frame rate is 30 frames per second with a resolution of $352 \times 288$, and the total number of frames is 300 .

The video is encoded using the Joint Scalable Video Model (JSVM) codec [14] with two-layer coarse-grained quality scalability (CGS) and hierarchical-B coding structure to provide quality and temporal scalability. The PSNR of the base layer and the enhancement layer are 36.83 and $39.16 \mathrm{~dB}$, respectively. The average bit-rate of the base layer and the entire video are 410.47 and $881.79 \mathrm{kbps}$, respectively. During the transmission, each network abstraction layer (NAL) unit is encapsulated in an RTP packet.

We compared the HH-8QAM and the HM(B/Q) (a traditional hierarchical modulation which superimposes a BPSK symbol with a QPSK symbol). The second group is HH12$\mathrm{QAM}$ and $\mathrm{HM}(\mathrm{Q} / \mathrm{Q})$ (a traditional hierarchical modulation which superimposes two QPSK symbols). Their BERs are given in Fig. 6.

We consider two cases: one is that the transmitter has the knowledge of channel SNR, and can configure the modulation accordingly, e.g., in an AWGN channel, or a fading channel whose CSI is promptly fed back to the transmitter; the other is that the transmitter only knows the average received SNR of a Rician fast-fading channel with the Rician factor of $8 \mathrm{~dB}$.

Given the importance of the base layer, we design the retransmission scheme accordingly: if the base layer in a 


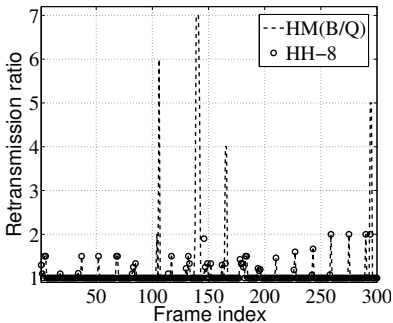

(a) Packet transmission time

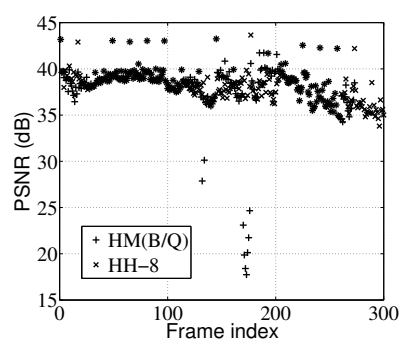

(b) Video PSNR
Fig. 8. HH-8QAM vs. HM(B/Q), one run, Rician fading

packet contains errors, a retransmission will be triggered until the base layer is received correctly or reaching the maximum (re-)transmission limit of 7 (similar to the IEEE 802.11 standard [13]).

For simplicity, the video data are fragmented to packets with the maximal packet size of 1500 bytes. For each setting, we repeated the simulation for 15 times with random seeds and calculated the average for comparison.

2) $H H-8 Q A M$ vs. $H M(B / Q)$ : Comparing HH-8QAM and $\mathrm{HM}(\mathrm{B} / \mathrm{Q})$, their average data rates are the same, one L1 bit and two L2 bits per symbol duration. We compared their performance using a $12.5 \mathrm{~dB}$ AWGN channel (for case 1) and a $14 \mathrm{~dB}$ Rician fading channel (for case 2). We used the strategy given in Sec. IV-A3 to configure the HH-8QAM and $\operatorname{HM}(B / Q)$, so their L2 raw BERs are in the order of $10^{-3}$.

In the AWGN case, since the channel is stable, we did not use link-layer retransmission for all modulations. At the receiver side, the un-decodable video frame ratios (due to packet losses for video base layer) for $\mathrm{HM}(\mathrm{B} / \mathrm{Q})$ and the proposed HH-8QAM are $9.4 \%$ and $1.5 \%$, respectively, and their corresponding PSNRs are 33.1 and $38.0 \mathrm{~dB}$, respectively. The results show that the proposed HH-8QAM can better protect L1 data and have a lower loss rate of L2 data, which results in a higher PSNR and a much lower frequency in video interruptions.

In the Rician fading case, we find the similar tendency, except that the performance of $\mathrm{L} 1$ are all degraded due to the random channel quality. To better protect L1 in this dynamic environment, the link layer retransmission scheme was adopted to allow up-to seven (re-)transmissions per packet. Also, the BER gap for the two layers for both HH-8QAM and HM(B/Q) are configured to be larger by enlarging the BER for $\mathrm{L} 2$ to $2 * 10^{-3}$.

Figure 8 (a) shows the packet transmission times (averaged for packets within the same frame) for a single run. Not only $\mathrm{HM}(\mathrm{B} / \mathrm{Q})$ needs more retransmissions, but also there are many cases that packets will be lost after the retry limit, which will bring a noticeable performance degradation for video, as shown in Fig. 8 (b).

Statistically, the average number of transmissions per packet is 1.09 and 1.12 , for $\mathrm{HH}-8 \mathrm{QAM}$ and $\mathrm{HM}(\mathrm{B} / \mathrm{Q})$. That is, $\mathrm{HM}(\mathrm{B} / \mathrm{Q})$ requires slightly more channel time than the proposed HH-8QAM. The average un-decodable video frame ratios for $\mathrm{HM}(\mathrm{B} / \mathrm{Q})$ and $\mathrm{HH}-8 \mathrm{QAM}$ are $3.8 \%$ and $1.9 \%$, respectively, and their corresponding PSNR are 35.4 and
$36.3 \mathrm{~dB}$, respectively.

Overall, the traditional $\mathrm{HM}(\mathrm{B} / \mathrm{Q})$ requires more retransmissions and has more video interruptions (due to more undecodable frames) which is unpleasant to users; the proposed HH-8QAM can outperform the existing HM to support video with a lower frequency of video interruptions, as well as a higher video PSNR.

\section{CONCLUSION}

In this paper, we have focused on the design and implementation of a non-binary communication system using hierarchical H-QAM, which can multiplex bits with trits to better support video applications and achieve a higher spectral and energy efficiency. The solution is simple to implement by a software upgrade to the existing system.

The proposed non-binary approach dramatically deviates from the conventional wisdom and it opens up many new research opportunities that beckon for further study. First, in this paper, we studied a limited number of hierarchical hexagonal modulation schemes as an example. Apparently, we have not fully explored the design space, and other schemes are possible for different application scenarios. For example, HH-QAM with denser constellation points are desirable for higher SNR scenarios. Second, it is possible to improve the constellation mapping to further reduce the BER and provide differentiated services, and the non-binary trellis coded modulation techniques can be a promising extension. In addition, we employed a $1 / 2$ rate $\mathrm{CC}$ code for trits, and more error correcting codes over $\mathrm{GF}(3)$ or hybrid $\mathrm{GF}(2)$ and $\mathrm{GF}(3)$ should be further investigated.

\section{REFERENCES}

[1] Index. Cisco visual networking index: Global mobile data traffic forecast update, 2011-2016, white paper, 2012.

[2] T. Cover. Broadcast channels. IEEE Trans. on Information Theory, 18(1):2-14, Jan. 1972.

[3] J. She, X. Yu, P. Ho, and E. Yang. A cross-layer design framework for robust IPTV services over IEEE 802.16 networks. IEEE JSAC 27(2):235-245, Feb. 2009.

[4] L. Cai, S. Xiang, Y. Luo, and J. Pan. Scalable modulation for video transmission in wireless networks. IEEE Trans. on Vehicular Technology, 60(9):4314 -4323, Nov. 2011.

[5] S. Han, J.M. Cioffi, and J. Lee. Tone injection with hexagonal constellation for peak-to-average power ratio reduction in OFDM. IEEE Communications Letters, 10(9):646 -648, Sept. 2006.

[6] Z. Yang, L. Cai, A. Gulliver, L. He, and J. Pan. Beyond the power of two: Hexagonal modulation and non-binary coding for wireless communication systems. Project Report, 2013.

[7] H. Schwarz, D. Marpe, and T. Wiegand. Overview of the scalable video coding extension of the H. 264/AVC standard. IEEE Trans. on Circuits and Systems for Video Technology, 17(9):1103-1120, 2007.

[8] M. Ettus. Ettus research, LLC. Online information on USRP board, available at http://www.ettus .com, 2008.

[9] Veljko Pejovic. OFDM Example. Available at http://www.cs.ucsb.edu/ $\sim$ veljko/downloads/ofdm_example.tar.gz, 2011.

[10] T. Koike and S. Yoshida. Space-time Trellis-coded ternary PSK for mobile communications. Electronics Letters, 40(16):1011-1012, 2004.

[11] B. Sklar. Digital communications, volume 2. Prentice Hall, 2001.

[12] R. Urbanke. On multiple-access communication. PhD thesis, Washington University, St. Louis, August 1995.

[13] M. Gast. 802.11 wireless networks: the definitive guide. O'Reilly Media, 2005.

[14] J. Reichel, H. Schwarz, and M. Wien. Joint scalable video model 11 (JSVM 11). Joint Video Team, Doc. JVT- X, 2007. 